\title{
Correction to "Pharmacogenomic Next-Generation DNA Sequencing: Lessons from the Identification and Functional Characterization of Variants of Unknown Significance in CYP2C9 and CYP2C19"
}

\begin{abstract}
In the above article [Devarajan S, Moon I, Ho M-F, Larson NB, Neavin DR, Moyer AM, Black JL, Bielinski SJ, Scherer SE, Wang L, Weinshilboum RM, and Reid JM (2019) Drug Metab Dispos 47 (4), 425-435; DOI: https://doi.org/10.1124/dmd.118.084269], a few mistakes were accidentally introduced during the preparation and review of the manuscript.

First, Authorship line, Dr. Sandhya Devarajan was incorrectly listed as the primary author for this work. Dr. Sandhya Devarajan and Ms. Irene Moon contributed equally to this work and now listed as co-first authors for the manuscript and a footnote added.

Second, Table 2, on page 428 , in the $4^{\text {th }}$ row titled 'Gene', the column 'rsID' should list 'Not present' instead of 'rs549167718'; column 'Validation' should be empty instead of '2'; column 'gnomAD Data Bank (Minor Allele Frequency)' should be 'Not recorded' instead of '4.099E- ${ }^{\text {} 6 \mathrm{a}}$ '. In the $5^{\text {th }}$ row titled 'Gene', the column 'gnomAD Data Bank (Minor Allele Frequency)' should have 'a' footnote reference instead of 'b'. In the footnote section below the table, footnote 'a without frameshift' should be deleted and footnote ' $b$ ' should be changed to ' $a$ '. The corrected table is provided below
\end{abstract}

TABLE 2

Data search results for $C Y P 2 C 9$ and $C Y P 2 C 19$ variants in gnomAD data bank.

The data base search results were obtained from gnomAD (http://gnomad.broadinstitute.org/) and dbSNP (https://www.ncbi.nlm.nih.gov/projects/SNP/) in August 2018.

\begin{tabular}{|c|c|c|c|c|}
\hline \multirow{2}{*}{ Gene } & \multirow{2}{*}{ cDNA Change } & \multicolumn{2}{|c|}{ dbSNP data bank } & \multirow{2}{*}{$\frac{\text { gnomAD Data Bank }}{\text { (Minor allele frequency) }}$} \\
\hline & & rsIDs & Validation & \\
\hline CYP2C9 & $218 \mathrm{C}>\mathrm{T}$ & rs762081829 & Not done & $5.69 \times 10^{-05}$ \\
\hline CYP2C9 & $229 \mathrm{C}>\mathrm{A}$ & Not present & & Not recorded \\
\hline CYP2C9 & $343 \mathrm{~A}>\mathrm{C}$ & rs 771237265 & 1 & $1.01 \times 10^{-04}$ \\
\hline CYP2C9 & 707delA & Not present & & Not Recorded \\
\hline CYP2C9 & $709 \mathrm{G}>\mathrm{C}$ & Not present & & Not recorded ${ }^{a}$ \\
\hline CYP2C9 & $791 \mathrm{~T}>\mathrm{C}$ & rs761895497 & 1 & $1.09 \times 10^{-05}$ \\
\hline CYP2C9 & $801 \mathrm{C}>\mathrm{T}$ & rs 149158426 & $1,2,3$ & $8.55 \times 10^{-04}$ \\
\hline CYP2C19 & $65 \mathrm{~A}>\mathrm{G}$ & rs 144928727 & 3 & $1.22 \times 10^{-05}$ \\
\hline CYP2C19 & $337 \mathrm{G}>\mathrm{A}$ & rs 145119820 & $1,2,3$ & $2.24 \times 10^{-04}$ \\
\hline CYP2C19 & $518 \mathrm{C}>\mathrm{T}$ & rs61311738 & $1,2,3$ & $4.66 \times 10^{-03}$ \\
\hline CYP2C19 & $556 \mathrm{C}>\mathrm{T}$ & rs 183701923 & $1,2,3$ & $9.74 \times 10^{-05}$ \\
\hline CYP2C19 & $557 \mathrm{G}>\mathrm{A}$ & rs 140278421 & $1,2,3$ & $1.08 \times 10^{-04}$ \\
\hline CYP2C19 & $578 \mathrm{~A}>\mathrm{G}$ & Not present & & $4.06 \times 10^{-06}$ \\
\hline CYP2C19 & $815 \mathrm{~A}>\mathrm{G}$ & Not present & & Not recorded \\
\hline
\end{tabular}

rsID, reference SNP identification; 1, Validated by frequency or genotype data (minor alleles were observed in at least two chromosomes); 2 , SNP has been sequenced in 1000 genome project; 3, Validated by multiple, independent submissions to the reference SNP cluster.

a (other mutations to Ile, Phe, Val and Ala recorded at this position).

Third, Results Section on page 428, right column, line seven ' $229 \mathrm{C}>\mathrm{A}$ and $709 \mathrm{G}>\mathrm{C}$ ' should be '229C $>$ A, 707delA and 709G $>$ C'. On page 429, left column, line 2, 'three of the identified CYP2C19 variants $(229 \mathrm{C}>\mathrm{A}, 709 \mathrm{G}>\mathrm{C}$ and $801 \mathrm{C}>\mathrm{T})$ ' should be 'five of the identified CYP2C9 variants $(218 \mathrm{C}>\mathrm{T}, 343 \mathrm{~A}>\mathrm{C}$, 707delA, 707_709delinsCC and 791T $>C$ ). On page 429, right column, line 2, '(65A $>\mathrm{G}, 337 \mathrm{G}>\mathrm{A}$, $578 \mathrm{~A}>\mathrm{G}$ and $815 \mathrm{~A}>\mathrm{G})$ should be $(518 \mathrm{C}>\mathrm{T}, 556 \mathrm{C}>\mathrm{T}, 557 \mathrm{G}>\mathrm{A}$ and $815 \mathrm{~A}>\mathrm{G})$.

Fourth, Table 4, on page 433, line one under the title, ' $518 C>C T, 556 C>C T$ ' should be ' $518 C>T, 556 C>T$ '.

The HTML and PDF versions of the article have been corrected.

The authors regret these errors and apologize for any inconvenience this may have caused. 